Ghanouni, A; Smith, SG; Halligan, S; Taylor, SA; Plumb, A; Boone, D; von Wagner, C; (2013) An interview study analysing patients' experiences and perceptions of non-laxative or full-laxative preparation with faecal tagging prior to CT colonography. Clin Radiol , 68 (5) pp. 472-478.

10.1016/i.crad.2012.10.012.

Article

\title{
An interview study analysing patients' experiences and perceptions of non-laxative or full-laxative preparation with faecal tagging prior to CT colonography
}

Alex Ghanouni ${ }^{1 b c e f g h}$ BSc MSc; Samuel G. Smith ${ }^{1 b c e g h}$ BSc MSc; Steve Halligan ${ }^{2 b c e g h}$ MD FRCR FRCP; Stuart A. Taylor ${ }^{2 b c e g h}$ MD MRCP FRCR; Andrew Plumb ${ }^{2 c e g h}$ MRCP FRCR; Darren Boone $^{\text {2bcegh }}$ BSc MRCS FRCR; Christian von Wagner ${ }^{\text {1abcetgh }}$ BSc PhD.
a. Guarantor of the integrity of the entire study
b. Study concepts and design
c. Literature research
d. Clinical studies (N/A)
e. Experimental studies / data analysis
f. Statistical analysis
g. Manuscript preparation
h. Manuscript editing

\section{Affiliations:}

${ }^{1}$ Department of Epidemiology and Public Health, University College London, London, UK ${ }^{2}$ Centre for Medical Imaging, University College London, London, UK

\author{
Corresponding author at: \\ Christian von Wagner \\ Health Behaviour Research Centre \\ Department of Epidemiology \& Public Health \\ UCL, Gower Street, London \\ WC1E 6BT, UK \\ Email:c.wagner@ucl.ac.uk \\ Tel: +44 (0)20 76791940 \\ Fax: $+44(0) 2076798354$
}

Word Count: 249 / 250 in abstract; 3376 / 4000 in main body

Conflicts of interest: None to declare.

Source of Funding: This article presents independent research commissioned by the National Institute for Health Research (NIHR) under its Programme Grants for Applied Research funding scheme (RP-PG-0407-10338). This work was undertaken at UCL and UCLH, who receive a proportion of funding from the NIHR Biomedical Research Centre funding scheme. The views 
expressed in this article are those of the authors and not necessarily those of the NHS, the NIHR or the Department of Health.

Acknowledgements: The authors thank Nichola Bell and Eva Macekova for assistance with inviting eligible participants and Elaine Atkins for assistance with recruitment.

Classifications: Gastrointestinal; CT

\begin{abstract}
Aim: This study compared patients' experiences of either non- or full-laxative bowel preparation with additional faecal tagging and subsequent CT colonography using in-depth interviews to elicit detailed responses.

Materials and Methods: Patients who received CT colonography after non- $(N=9)$ or full-laxative $(\mathrm{N}=9)$ preparation participated in a semi-structured telephone interview at least two days after investigation. Full-laxative preparation consisted of magnesium citrate and sodium picosulphate administered at home (or polyethylene glycol, if contraindicated), followed by hospital-based faecal tagging with iohexol. Non-laxative preparation was home-based barium sulphate for faecal tagging. Interviews were transcribed and thematically analysed to identify recurrent themes on patients' perceptions and experiences.
\end{abstract}

Results: Experiences of full-laxative preparation were usually negative and characterised by pretest diarrhoea that caused significant interference with daily routine. Post-test flatus was common. Non-laxative preparation was well-tolerated; patients reported no or minimal changes to bowel habit and rapid return to daily routine. Patients reported worry and uncertainty about the purpose of hospital-based faecal tagging. This also added burden from waiting before testing.

Conclusion: Patients' responses supported previous findings that non-laxative preparation is more acceptable than full-laxative preparation but both can be improved. Faecal tagging used in combination with laxative preparation is poorly understood, adding burden and worry. Home-based non-laxative preparation is also poorly understood and patients require better information on the purpose and mechanism in order to give fully informed consent. This may also optimise adherence to instructions. Allowing home-based self-administration of all types of preparation would prevent waiting before testing.

\title{
Introduction
}

CT colonography (CTC) is replacing barium enema as the radiologic investigation of choice for diagnosis of colorectal cancer (CRC) and polyps in both symptomatic and screening populations. International consensus guidelines recommend CTC be performed following full cathartic bowel preparation, preferably with oral contrast to 'tag' colonic residue ${ }^{1-3}$. Cathartic preparation with oral tagging is used widely since it is designed to maximise test accuracy. However, many patients perceive laxative bowel preparation as the worst aspect of the test ${ }^{4,5}$ and the addition of 'faecal tagging' may add additional burden. Quantitative studies of acceptability have found non-laxative preparation to be equivalent or superior to full-laxative preparation in terms of its acceptability ${ }^{6-10}$. However, although non-laxative CTC is likely to be better tolerated, its diagnostic accuracy compared to full-laxative studies is currently uncertain and so this preparation is generally reserved for patients considered unfit for full catharsis ${ }^{11}$.

Uptake of screening with full-laxative CTC is low (approximately $22 \%$ after a first invitation ${ }^{12}$ ). A recent randomized study found non-laxative CTC achieved higher screening uptake than fulllaxative colonoscopy (34\% vs. $22 \%$ ), supporting the argument that non-laxative preparation may 
have wider applications ${ }^{13}$. Comprehensive evaluation of both preparations is warranted in order to make full comparisons. Furthermore, both types of preparations should be as acceptable as possible, whenever indicated, and so evaluations should also aim to discover where there is scope for improvement. Previous evaluations of patients' experiences using questionnaires are limited to measuring specific aspects, such as the nature and severity of side effects. However, bowel preparation is multifactorial, involving factors such as interference with daily life and possible concerns at the hospital before the test so it is important to explore patients' views in more depth. Moreover, many studies of oral faecal tagging predate its widespread implementation, meaning that little is known regarding patients' opinions of this aspect of their diagnostic test experience when delivered as part of routine clinical practice.

The aim of the present study was to evaluate and compare patients' understanding, perceptions and experiences with the two types of preparations and gain a more complete understanding by using semi-structured interviews in order to elicit detailed responses.

\section{Materials and Methods}

\section{Design and participants}

Following full ethical review and receiving a favourable opinion from an NHS Research Ethics Committee, a consecutive sample of patients scheduled to attend a local radiology department at a large, urban teaching hospital was identified using the hospital booking system. Patients were due to undergo CTC, following either full-laxative or non-laxative preparation. Prior to CTC, a hospital administrator mailed an information sheet and consent form to eligible participants, inviting them to take part in a semi-structured telephone interview with a researcher (BLIND FOR REVIEW). Patients were met prior to CTC by the researcher to discuss the study, obtain informed consent from patients willing to take part and schedule a telephone interview. Interviews were conducted at least two days later and (based on feedback from the Research Ethics Committee) participants were offered 10GBP for taking part. Patients were excluded if they had not completed prescribed bowel preparation, were unable to speak English, or were otherwise unable to consent.

\section{Procedures}

\section{Preparation}

Protocols for bowel preparation are described in Figure 1. Local practice is for the referring clinician to prescribe full-laxative bowel preparation using combined magnesium citrate and sodium picosulphate preparation (Picolax, Ferring Pharmaceuticals, Slough, UK) unless contra-indicated (e.g. renal or cardiac failure), in which case polyethylene glycol (Klean-Prep, Norgine Pharmaceuticals Ltd., Uxbridge, UK) is given. For faecal tagging, patients subsequently receive $100 \mathrm{ml}$ oral iohexol (Omnipaque, Bayer Healthcare, Newbury, UK) in the radiology department, two hours prior to CTC.

Patients considered too frail for any purgation are prescribed barium sulphate $4.9 \%$ weight/volume suspension (E-Z-CAT, Bracco, Lake Success, NY, USA).

\section{CT colonography}

A standardised CTC protocol was performed in all patients. $20 \mathrm{mg}$ of hyoscine-N-butylbromide (Buscopan, Boehringer Ingelheim) and 100mls of iohexol (Omnipaque 350, Amersham Health) were given intravenously unless contraindicated. $\mathrm{CO}_{2}$ insufflation was achieved by an experienced practitioner using a thin rectal balloon catheter and automated insufflator $\left(\mathrm{PROTOCO}_{2} \mathrm{~L}, \mathrm{E}-\mathrm{Z}-\mathrm{EM}\right.$, Bracco, Lake Success, NY, USA); 2-3I $\mathrm{CO}_{2}$ on average. 


\section{Measures}

An interview topic guide was developed by three health psychology researchers, BLIND FOR REVIEW, following consultation with three radiologists (with experience of over 1000 CTC examinations each; BLIND FOR REVIEW) and literature review ${ }^{7-10,14}$. Open-ended questions were presented in a flexible order (see Table 1 for key questions). Interviews were audio recorded for subsequent analysis.

\section{Analysis}

We used a technique known as "thematic analysis" 15: Recordings were transcribed and analysed by two health psychology researchers (BLIND FOR REVIEW) using software designed for qualitative research (NVivo 9 for Windows, QSR International). Transcripts were read repeatedly and participants' quotes extracted and categorised based on a framework that corresponded to the order in which events happened to participants (i.e. preparation, the hospital visit, after the test). After this initial categorisation, each section was explored to identify sub-themes. These were considered in relation to each other to determine whether they were distinct or could be grouped together. Final themes were reviewed by radiologists (BLIND FOR REVIEW) to provide important additional context. This process allows researchers to form a cohesive interpretation of a complex, rich dataset.

\section{Results}

\section{Sample Characteristics}

Two patients receiving full-laxative preparation did not receive oral iohexol for faecal tagging in hospital and one patient receiving non-laxative preparation did. These patients were excluded from subsequent analysis. Approximately 105 invitations were sent out. The Research Ethics Committee reviewing this study estimated that a sample of 15 participants per group would be appropriate in order to obtain sufficient data. However, following input from an experienced qualitative researcher who read interview transcripts and preliminary themes, we estimated that data saturation had occurred after nine analysable interviews per group (i.e. those patients receiving CTC within the standard preparation protocol). Additional data were considered unlikely to develop our themes further but would result in burden for subsequent participants ${ }^{16}$ and so (following ethical approval) recruitment was halted at this point. All 18 patients completed CTC after full-laxative preparation (polyethylene glycol, PEG or sodium picosulphate and magnesium citrate, SPMC; mean age $=65$ years; $67 \%$ female) or non-laxative preparation (barium sulphate, $\mathrm{Ba}_{2} \mathrm{SO}_{4} ;$ mean age $=74$ years, 67\% female). Patients frequently reported prior experience with gastrointestinal imaging, including five patients who had undergone colonoscopy, two other patients who had undergone CTC, and a further patient who had experienced flexible sigmoidoscopy.

Referral reasons (as reported by patients) for undergoing CTC with non-laxative preparation included bowel-related symptoms such as abdominal pain, diarrhoea or rectal bleeding $(\mathrm{N}=4)$, positive FOBt $(\mathrm{N}=3)$ and as follow-up after other investigations $(\mathrm{N}=2)$. Patients undergoing fulllaxative preparation reported being referred for bowel-related symptoms $(\mathrm{N}=3)$, follow-up testing $(\mathrm{N}$ $=5)$ and positive FOBt $(\mathrm{N}=1)$. Interviews were carried out between 3 and 34 days after patients' investigations (median $=5$ days). Several patients reported that they were currently waiting for their results $(\mathrm{N}=15)$ and no patient reported having received them at the time of the interview. Local protocol for follow-up leads to results being provided to patients approximately 14 days after their investigations, meaning that only one of the remaining three patients might have received them by the time they were interviewed.

Figure 2 presents a summary of themes associated with patient experience. 


\section{Experience with dietary changes}

Full-laxative.

Patients often reported dietary changes as a hardship ("The main thing is that I got [very] hungry, with all the consequences that has in terms of feeling tired and possibly a bit irritable and distracted"; male, 58, SPMC). Patients also struggled with the high volume of fluid intake and with eating the recommended foods ("There's no way that l'd eat just plain fish with potatoes or rice or plain chicken...You know, I just think that's gross...You've got to have all your spices with it"; female, 57, SPMC).

Non-laxative.

Most patients undergoing non-laxative preparation found the dietary changes to be tolerable ("It was alright...I didn't have any complaints with the diet part of it"; female, 74, $\mathrm{Ba}_{2} \mathrm{SO}_{4}$ ) and some reported no problems. Dietary changes were often perceived as manageable due to their short duration ("You know, two days, that's not a problem, is it?"; female, 76, $\mathrm{Ba}_{2} \mathrm{SO}_{4}$ ). However, some patients did report a degree of hunger or other problems with specific aspects of the diet ("Drinking tea without milk in it...that's pretty awful"; female, $76, \mathrm{Ba}_{2} \mathrm{SO}_{4}$ ).

\section{Experience of the effects of medicine at home}

\section{Full-laxative.}

Most patients reported highly negative experiences with the effects of full-laxative solution, including "the most appalling, explosive diarrhoea" (female, $79, \mathrm{Ba}_{2} \mathrm{SO}_{4}$, describing a previous CTC with fulllaxative preparation). One patient recalled that "every three minutes, I have to go to the toilet. Oh, it was horrible" (male, 66, PEG). Laxative effects also interfered considerably with patients' daily routine ("Pretty awful, I didn't go to work that day 'cause I was running to the toilet"; female, 69, SPMC). A number of other physical effects were mentioned, including abdominal bloating and sleep interference. There were few exceptions to these negative experiences ("It wasn't a bad experience, I really shouldn't say how horrible it is, it really wasn't that bad"; female, 63, SPMC).

\section{Non-laxative.}

Experiences were less negative in this group compared to patients undergoing full-laxative preparation ("I was normal, I was surprised it was normal. Didn't do anything like diarrhoea or anything"; male, $63, \mathrm{Ba}_{2} \mathrm{SO}_{4}$ ). Some reported no difficulties ("there was nothing to it...If they'd said to me, you've got to do this for a week, it would have been no problem"; male, 67, $\mathrm{Ba}_{2} \mathrm{SO}_{4}$ ). Patients who had experience of both preparations (i.e. patients with prior experience with colonoscopy or CTC) stated a preference for non-laxatives ("[with SPMC]...you might as well just sit in a toilet...I had no problem [with $\mathrm{Ba}_{2} \mathrm{SO}_{4}$ ]"; male, 67, $\mathrm{Ba}_{2} \mathrm{SO}_{4}$ ).

Others described looser bowel motions ("The [information materials] talk about the stools may be paler and I didn't notice that particularly but they were a little looser"; female, $79, \mathrm{Ba}_{2} \mathrm{SO}_{4}$ ). However, this was not always attributed to the preparation ("A bit of a puzzle because I don't know whether it was responsible for me having bad diarrhoea or not"; female, 77, $\mathrm{Ba}_{2} \mathrm{SO}_{4}$ ).

\section{Patient experiences before and during the test}

\section{Full-laxative.}

Patients frequently commented on the "very boring wait" (male, 76, SPMC) that was necessary after taking iohexol, with one calling it "a waste of my time" (female, 63, SPMC). This patient also questioned why the drink was not available earlier ("Why couldn't they] provide me with that drink 
before I got there and then show up at 10.30 instead of 9.30?'). Two patients reported feeling uncomfortable while waiting due to being "tired because of lack of sleep, lack of food and dehydrated" (male, 66, PEG).

\section{Non-laxative.}

Patients undergoing non-laxative preparation reported few issues of discomfort before CTC. The wait before CTC was tolerated comparatively well and two patients reported few to no problems ("Bit of waiting but not too much"; female, $80, \mathrm{Ba}_{2} \mathrm{SO}_{4}$ ).

Both groups made similar comments with respect to CTC itself. Both reported having concerns prior to testing regarding rectal catheterisation, breath-holding and turning. However, patients usually found that these aspects were not as difficult or unpleasant as expected ("I was a bit worried that I wasn't going to be able to hold [my] breath for that long...but I seemed to do alright...It didn't last long, I was expecting something to last very, very long but it wasn't 5 minutes"; female, 69, SPMC). Both groups reported insufflation to be the most uncomfortable aspect of the test itself, although discomfort was mild and pain was rare.

\section{Immediate and longer-term after-effects}

\section{Full-laxative.}

The most commonly reported after-effect was difficulty with passing flatus, which several patients experienced ("They told me not to go home until the gas come out...It took more than half an hour, I just couldn't get it out"; male, 66, PEG). Patients also reported experiencing flatus until the next day or longer although this did not appear to cause significant concerns or discomfort. Some patients reported continued disruption to their usual routine due to feeling tired, dehydrated or unable to eat. Finally, patients often reported a continued change in bowel habit with increased frequency and looseness ("Experienced a little bit of gas and a little bit of diarrhoea"; female, 63, SPMC).

\section{Non-laxative.}

Patients in this group reported a more rapid recovery. Fewer reported difficulties passing flatus ("Just went to the toilet for a few minutes, passed some wind, then just go home"; male, 63, $\mathrm{Ba}_{2} \mathrm{SO}_{4}$ ) and patients did not report continued changes in bowel habit after the test. Patients also seemed to be more likely to return to their usual routines immediately after the test. However, two patients reported that they did not return to a normal diet immediately ("We didn't get a breakfast and I wasn't able to manage that"; female, $74, \mathrm{Ba}_{2} \mathrm{SO}_{4}$ ). This patient also felt "a bit disoriented" and another "felt dizzy" (female, $82, \mathrm{Ba}_{2} \mathrm{SO}_{4}$ ). Patients were comparable to the full-laxative preparation group in terms of reported tiredness and loss of energy.

\section{Perceptions of the purpose of bowel preparations}

\section{Full-laxative.}

Most patients felt confident that they understood the purpose of full-laxative preparation, often reporting it to be for 'clearing out' the bowel. Some patients expressed a lay understanding of sensitivity ("[The bowels have to be clean, otherwise when [the clinicians] take the scan...[they] won't be able to see clearly... There's no solids there otherwise [they] won't be able to see what's wrong with it"; male, 66, PEG). Others mentioned specificity ("Clear the bowels so that nothing would show up on the scan that...might look like a foreign object or something"; female, 69, SPMC). One patient also perceived it as necessary for insufflation ("Because they wanted my bowels to be empty...to pump air and see what's happening inside me"; female, 67, SPMC). 
However, patients had a much poorer understanding of the purpose of faecal tagging administered in hospital. Some patients receiving this recalled being worried and uncertain about the purpose (" $A$ [staff member], she gave me a medical drink and she said go out and come back in...two hours' time and you do a test...I had no idea what it's for"; male, 66, PEG). Patients were also concerned about the possibility of an allergic reaction to iodine.

Non-laxative.

As was observed in the full-laxative group, patients had poor understanding of the purpose and mechanism of faecal tagging. Some patients in this group also described the purpose in terms of 'clearing out' but were notably less confident in their understanding that those taking full-laxative preparation at home ("I was still thinking, I wonder what it does do then"; male, 67, $\mathrm{Ba}_{2} \mathrm{SO}_{4}$ ). However, this was rarely a cause for concern and patients were willing to adhere to a clinical recommendation without much consideration ("you don't go into such details, if they demand it from you, you do it"; female, 82, $\mathrm{Ba}_{2} \mathrm{SO}_{4}$ ).

\section{Discussion}

In this study of patient perceptions and experiences with two commonly used methods of bowel preparation, we found that patients' knowledge and understanding of faecal tagging preparations (whether administered at home or in hospital) was poor compared to full-laxative preparation, the purpose of which was regarded to be "common sense". Patients were willing to adhere to clinical instructions for faecal tagging but expressed concern about its purpose and mechanism. Patients undergoing full-laxative preparation reported significant negative physical effects (in terms of bowel habit, mood and appetite) and disruption to daily routine whereas the effects of non-laxative preparation were generally mild. These latter findings support and decompose those of previous quantitative studies that find non-laxative preparation leads to greater "burden", is "(much) better", less "burdensome", "preferred" and leads to greater "global discomfort" compared to full-laxative preparation ${ }^{6-10}$ but do not qualify how these differences are experienced by patients.

The strengths of this study lie in the use of a semi-structured interview design that allowed us to compare experiences between patient groups in depth and so identify previously unconsidered aspects. The sample size was sufficient to achieve a thorough understanding of patients' experiences and so these findings can be applied to future research and practice.

We found that patients primarily understood the purpose of non-laxative preparation (administered at home) in terms of emptying the bowel. This is understandable since the dietary restrictions do remove faeces and several patients reported diarrhoea (possibly due to the inclusion of sorbitol). Although it is reassuring that patients were not anxious regarding their lack of understanding of home-based non-laxative preparation, there was more concern about not knowing the purpose of hospital-based oral contrast, partly due to the stated risks. Patients' understanding of the purpose of this specific part of full-laxative preparation was particularly poor when it had no noticeable effects. It is important that patients have enough knowledge of the purpose of prescribed medications to give fully informed consent. This would not require patients to become subject matter experts but it is appropriate and achievable to ensure that patients have at least a cursory understanding of the reason behind being given any medication. In addition, patients' concerns about oral contrast might be ameliorated quite simply by providing more information on the reasons why oral contrast is prescribed ${ }^{17}$.

We found that patients taking non-laxative preparation at home were willing to comply with instructions, despite poor understanding of the purpose. However, lack of understanding of the purpose of faecal tagging preparation at home raises the possibility of poorer adherence to instructions and contingent negative effects on preparation quality ${ }^{18}$. For example, patients may not 
recognise why it is recommended that barium sulphate be taken with food unless they understand that it tags faeces, which may mean they take it at other times.

Although hospital-based faecal tagging with oral contrast has been found to be acceptable in terms of both discomfort and side-effects ${ }^{19}$, patients in our study reported dissatisfaction with the necessary wait before CTC. Allowing patients to self-administer all preparation at home may improve acceptability but this would need to be balanced against the theoretical risks of allergic reaction, potential laxative effects, and the need to administer within three hours of CTC. Where home-based administration is considered impractical, patients should be provided with information regarding why hospital administration is necessary.

Both groups reported poor understanding of some aspects of their preparation, raising questions over contributing factors. The most likely possibility is that information materials did not include relevant information and so may be failing to meet patients' needs. Appendices 1-4. show extracts of the information provided at the recruiting hospital, demonstrating that the purpose of $\mathrm{Ba}_{2} \mathrm{SO}_{4}$ is not stated accurately. Furthermore, the purpose of oral contrast is described as "[helping] better understand the results of the scan" which patients may find unclear. Patients may also find it difficult to assimilate information provided on bowel preparation since they are already considering a large amount of information relating to other aspects of the investigation. Patient information should be tested with users in order to remove these possibilities and future research may assess whether poor understanding of the purpose and mechanisms behind non-laxative preparation adversely affects preparation quality.

This study has limitations. Two full-laxative preparations were used and there may have been subtle differences between them. For example, we did not observe differences in acceptability with respect to the volume of liquid that patients were advised to drink although this has previously been found to affect patients' experiences ${ }^{20,21}$. In addition, this study took place in a single centre and findings may not generalise fully to hospitals that organise their CTC services differently, particularly with respect to hospital-based faecal tagging and information materials. However, the study sample was recruited from an ethnically and socioeconomically diverse area and patients varied in terms of referral reasons. On average, patients receiving non-laxative preparation were older than those receiving laxative preparation and so more likely to have co-morbidities, consistent with standards of how these preparations are prescribed. Our higher order findings on patients' experience were consistent with previous literature, further supporting the generalisability of this sample.

In conclusion, although non-laxative preparation was more acceptable than full-laxative preparation, this study identified several possible methods of improving both. There is a need for more effective information provision for both home-based, non-laxative bowel preparation, and oral contrast when administered for faecal tagging following full-laxative preparation. Future research may investigate the best approach in helping patients understand bowel preparation and assess the extent to which this could influence preparation quality.

\section{References}

1. Burling D. CT colonography standards. Clinical Radiology. 2010;65(6):474-80.

2. Taylor SA, Laghi A, Lefere P, Halligan S, Stoker J. European Society of Gastrointestinal and Abdominal Radiology (ESGAR): Consensus statement on CT colonography. European Radiology. 2007;17(2):575-9.

3. Barish MA, Soto JA, Ferrucci JT. Consensus on current clinical practice of virtual colonoscopy. American Journal of Roentgenology. 2005;184(3):786-92. 
4. von Wagner C, Smith S, Halligan S, Ghanouni A, Power E, Lilford RJ, et al. Patient acceptability of CT colonography compared with double contrast barium enema: Results from a multicentre randomised controlled trial of symptomatic patients. European Radiology. 2011; 21(10):2046-55.

5. Ristvedt SL, McFarland EG, Weinstock LB, Thyssen EP. Patient preferences for CT colonography, conventional colonoscopy, and bowel preparation. The American Journal of Gastroenterology. 2003;98(3):578-85.

6. Jensch S, Bipat $S$, Peringa J, de Vries AH, Heutinck A, Dekker $E$, et al. CT colonography with limited bowel preparation: prospective assessment of patient experience and preference in comparison to optical colonoscopy with cathartic bowel preparation. European Radiology. 2010;20(1):146-56.

7. Taylor SA, Slater A, Burling DN, Tam E, Greenhalgh R, Gartner L, et al. CT colonography: optimisation, diagnostic performance and patient acceptability of reduced-laxative regimens using barium-based faecal tagging. European Radiology. 2008;18(1):32-42.

8. Florie J, van Gelder RE, Schutter MP, van Randen A, Venema HW, de Jager S, et al. Feasibility study of computed tomography colonography using limited bowel preparation at normal and low-dose levels study. European Radiology. 2007;17(12):3112-22.

9. Iannaccone R, Laghi A, Catalano C, Mangiapane F, Lamazza A, Schillaci A, et al. Computed tomographic colonography without cathartic preparation for the detection of colorectal polyps. Gastroenterology. 2004;127(5):1300-11.

10. Lefere PA, Gryspeerdt SS, Dewyspelaere J, Baekelandt M, Van Holsbeeck BG. Dietary fecal tagging as a cleansing method before CT colonography: Initial results Polyp detection and patient acceptance. Radiology. 2002;224(2):393-403.

11. lafrate F, Hassan C, Zullo A, Stagnitti A, Ferrari R, Spagnuolo A, et al. CT colonography with reduced bowel preparation after incomplete colonoscopy in the elderly. European Radiology. 2008;18(7):1385-95.12. von Wagner C, Halligan S, Atkin WS, Lilford RJ, Morton D, Wardle J. Choosing between CT colonography and colonoscopy in the diagnostic context: a qualitative study of influences on patient preferences. Health Expectations. 2009;12(1):18-26.

12. Khalid-de Bakker C, Jonkers D, Smits K, Mesters I, Masclee A, Stockbrügger R. Participation in colorectal cancer screening trials after first-time invitation: a systematic review. Endoscopy. 2011;43(12):1059-86.

13. Stoop EM, de Haan MC, de Wijkerslooth TR, Bossuyt PM, van Ballegooijen M, Nio $\mathrm{CY}$, et al. Participation and yield of colonoscopy versus non-cathartic CT colonography in population-based screening for colorectal cancer: a randomised controlled trial. The Lancet Oncology. 2011;2045(11):1-10. 
14. Zalis ME, Perumpilichira JJ, Magee C, Kohlberg G, Hahn PF. Tagging-based, electronically cleansed CT colonograpy: Evaluation of patient comfort and image readability. Radiology. 2006;239(1):149-59.

15. Braun V, Clarke V. Using thematic analysis in psychology. Qualitative Research in Psychology. 2006;3(2):77-101.

16. Glaser BG, Strauss A. The discovery of grounded theory: Strategies for qualitative research. Chicago, IL: Aldine; 1967.

17. Betti S, Sironi A, Saino G, Ricci C, Bonavina L. Effect of the informed consent process on anxiety and comprehension of patients undergoing esophageal and gastrointestinal surgery. Journal of Gastrointestinal Surgery. 2011;15(6):922-7.

18. Spiegel BM, Talley J, Shekelle P, Agarwal N, Snyder B, Bolus R, et al. Development and validation of a novel patient educational booklet to enhance colonoscopy preparation. American Journal of Gastroenterology. 2011;106(5): 875-883.

19. Neri E, Turini F, Cerri F, Vagli P, Bartolozzi C. CT colonography: same-day tagging regimen with iodixanol and reduced cathartic preparation. Abdominal Imaging. 2009;34(5):642-7.

20. Kolts BE, Lyles WE, Achem SR, Burton L, Geller AJ, MacMath T. A comparison of the effectiveness and patient tolerance of oral sodium phosphate, castor oil, and standard electrolyte lavage for colonoscopy or sigmoidoscopy preparation. The American Journal of Gastroenterology. 1993;88(8):1218-23.

21. Vanner SJ, MacDonald PH, Paterson WG, Prentice RS, Da Costa LR, Beck IT. A randomized prospective trial comparing oral sodium phosphate with standard polyethylene glycol-based lavage solution (Golytely) in the preparation of patients for colonoscopy. The American Journal of Gastroenterology. 1990;85(4):422-77. 
a) Full-laxative preparation

a)i) Polyethylene glycol (Klean-Prep) lohexol (Omnipaque)

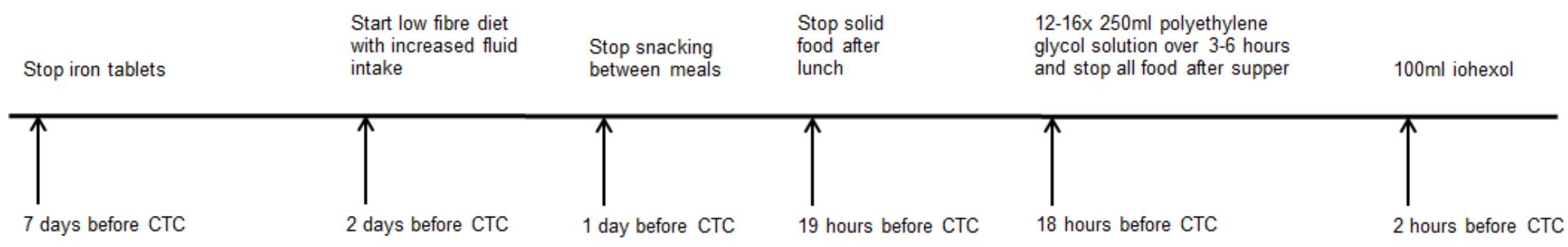

a)ii) Sodium picosulphate and magensium citrate (Picolax) lohexol (Omnipaque)

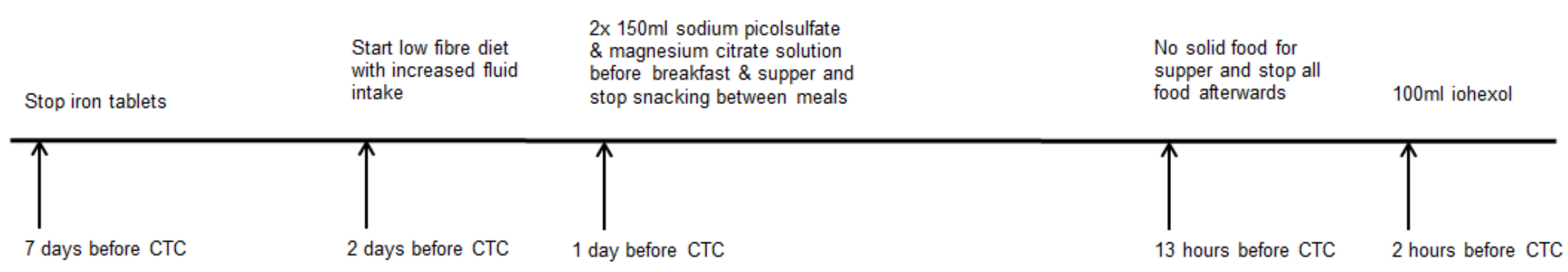

b) Non-laxative preparation (barium sulphate; E-Z-CAT)

Stop iron tablets




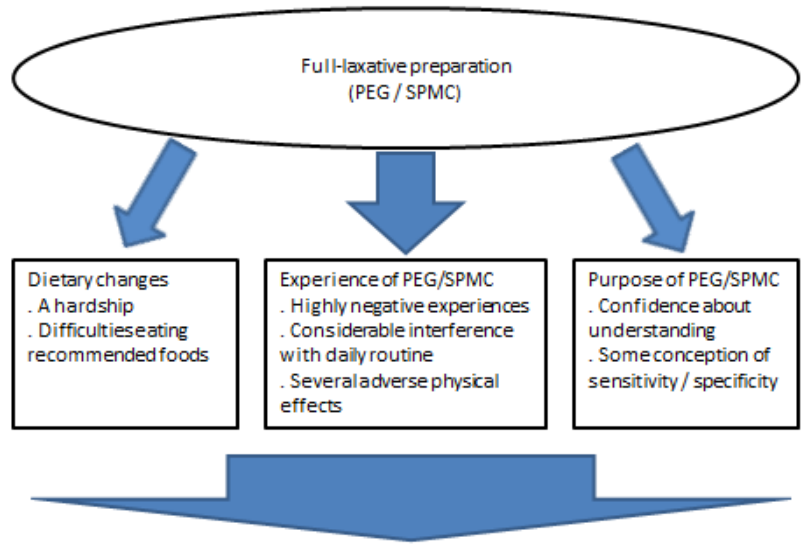

\section{Before thetest \\ Long, uncomfortablewaiting time after taking iohexol \\ Lack of understanding about the purpose of iohexol \\ Concerns about the test procedure}

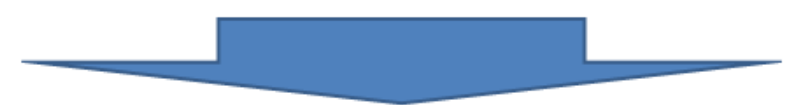

\section{During the test}

Concerns were not as problematic as anticipated

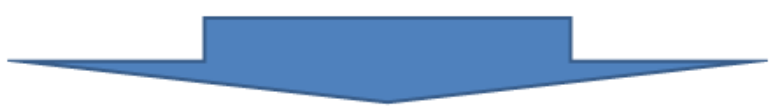

\section{After the test}

Difficulty passing flatus

Somecontinued disruption to daily routine

Somecontinued adverse physical effects

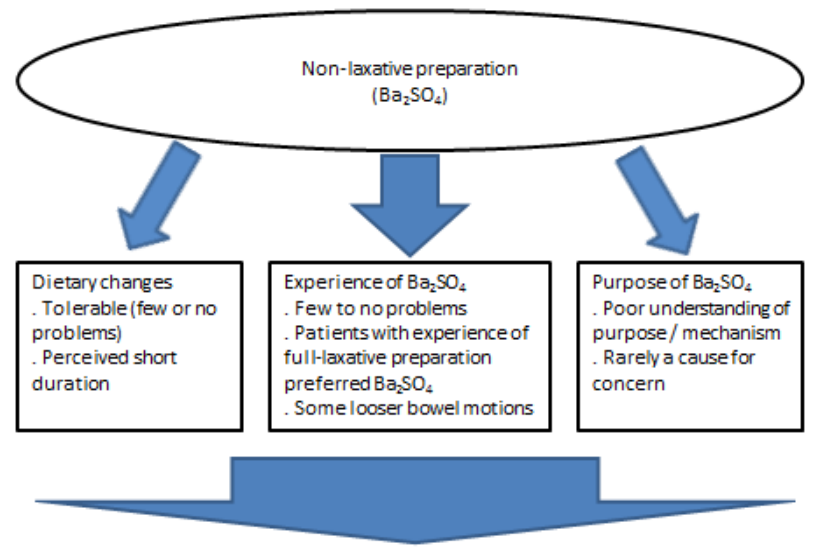

Before thetest

Waiting was well-tolerated and not uncomfortable

Concerns about the test procedure

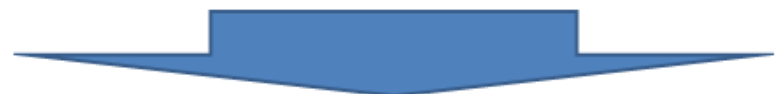

During the test

. Concernswere not as problematic as anticipated

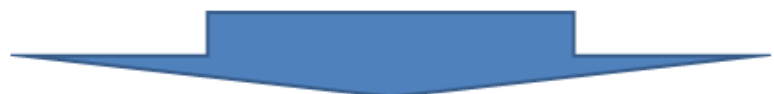

After the test.

Few difficulties passing flatus

a irly rapid recovery

Little continued disruption to daily routine

Few continued adverse physical effects 